\title{
Application of 3D printing rapid prototyping-assisted percutaneous fixation in the treatment of intertrochanteric fracture
}

\author{
SHENG-NAI ZHENG ${ }^{1,2^{*}}$, QING-QIANG YAO ${ }^{1-3^{*}}$, FENG-YONG MAO ${ }^{1,2}$, \\ PENG-FEI ZHENG ${ }^{3}$, SHU-CHANG TIAN ${ }^{1}$, JIA-YI LI ${ }^{1,2}$, YI-FAN YU ${ }^{1,2}$, SHUAI LIU ${ }^{1}$, \\ JIN ZHOU ${ }^{1}$, JUN HU ${ }^{1,2}$, YAN XU ${ }^{2,3}, \mathrm{KAITANG}^{3}$, YUE LOU ${ }^{3}$ and LI-MING WANG ${ }^{1-3}$ \\ ${ }^{1}$ Department of Orthopedic Surgery, ${ }^{2}$ Clinical Medical Center of Orthopedic Surgery; ${ }^{3}$ Digital Medicine Institute, \\ Nanjing Medical University, Nanjing Hospital, Nanjing, Jiangsu 210006, P.R. China
}

Received January 14, 2017; Accepted April 21, 2017

DOI: $10.3892 /$ etm.2017.4991

\begin{abstract}
The aim of the present study was to investigate the application of $3 \mathrm{D}$ printing (3DP) rapid prototyping (RP) technique-assisted percutaneous fixation in the treatment of femoral intertrochanteric fracture (ITF) using proximal femoral nail anti-rotation (PFNA). A total of 39 patients with unstable ITF were included in the current study. Patients were divided into two groups: 19 patients were examined using computed tomography scanning and underwent PFNA with SDP-RP whereas the other 20 patients underwent conventional PFNA treatment. Anatomical data were converted from the Digital Imaging and Communications in Medicine format to the stereolithography format using M3D software. The 3DP-RP model was established using the fused deposition modeling technique and the length and diameter of the main screw blade was measured during the simulation. The postoperative femoral neck-shaft angle (NSA), surgery duration, intraoperative and postoperative blood loss, and the duration of hospital stay were recorded and compared with the corresponding values in conventional surgery. No significant differences were observed in mean PFNA size between the implants used and the preoperative planning estimates. It was demonstrated that the 3DP-RP assisted
\end{abstract}

Correspondence to: Dr Li-Ming Wang, Department of Orthopedic Surgery, Nanjing Medical University, Nanjing Hospital, 68 Changle Road, Nanjing, Jiangsu 210006, P.R. China

E-mail: wanglimingnj@gmail.com

"Contributed equally

Abbreviations: 3DP, three-dimensional printing; RP, rapid prototyping; ITF, intertrochanteric fracture; PFNA, proximal femoral nail anti-rotation; CT, computed tomography; STL, stereolithography file; TAD, tip-apex distance

Key words: femoral intertrochanteric fracture, proximal femoral nail anti-rotation, $3 \mathrm{D}$ printing rapid prototyping procedure resulted in more effective reduction of the NSA. Furthermore, patients undergoing 3DP-RP experienced a significant reduction in duration of surgery $(\mathrm{P}<0.01)$, as well as reductions in intraoperative $(\mathrm{P}=0.02)$ and postoperative $(\mathrm{P}=0.03)$ blood loss, compared with conventional surgery. At 6 months post-surgery, no cases of hip varus/vague deformities or implant failure were observed in patients that underwent either the 3DP-RP-assisted or conventional procedure. The results of the present study suggest that the 3DP-RP technique is able to create an accurate model of the ITF, which facilitates surgical planning and fracture reduction, thus improving the efficiency of PFNA surgery for ITFs.

\section{Introduction}

At present, hip fracture is one of the most prevalent health problems in elderly patients with osteoporosis due to medical advances and increased life expectancy, and can lead to dysfunction and a $10 \%$ morbidity during the first month after injury (1). Intertrochanteric fractures (ITF) are one of the most common fractures of the hip and primarily occur in elderly people with osteoporosis (2). Conservative treatments of ITF are associated with high complication and mortality rates in the elderly; therefore surgery has become the preferred method of treatment (3). A number of studies have reported that intramedullary fixation has advantages over extramedullary fixation in ITF treatment, as it is minimally invasive, has a shorter surgical duration and is mechanically superior (4).

Compared with dynamic hip screw and Gamma nail, the use of proximal femoral nail anti-rotation (PFNA) may be biomechanically advantageous in the treatment of ITF, with a relatively shorter arm of force and a higher anti-torsion biomechanical performance $(5,6)$. PFNA is an intramedullary device that includes a helical blade inserted by impaction; the resulting bone compaction around the blade helps to prevent rotation and varus collapse (7). Previous studies have reported low complication rates and satisfactory results following the use of PFNA (8-10), thus it has become the most commonly used method of treating unstable ITF (11-13). However this 
technique is technically demanding and takes a long time to learn. The complex three-dimensional anatomy of bone dislocation is difficult to assess, which may lead to a prolonged period of traction reduction and radiation exposure (14). Furthermore, the severe displacement of ITF fragments may make closed reduction and internal fixation prone to varus/vague deformities (15).

In recent years, there have been rapid advances in the application of digital medicine and 3D printing rapid prototyping (3DP-RP) technology in orthopedic surgery (16). Using 3D-RP, it is possible to accurately recreate a complex anatomical structure to use for targeted preoperative planning (17). The aim of the present study was to prospectively describe the process of generating solid anatomical models of ITF structures that may be used to facilitate preoperative planning for PFNA internal fixation surgery.

\section{Materials and methods}

Patients. Between August 2014 and May 2016, 39 patients with unstable ITF were treated at the Nangjing Medical University, Nanjing Hospital and were divided into two groups based on patient preference. The 3DP-RP assisted surgery group consisted of 19 patients (11 men and 8 women; mean age of 66.2 years; age range, 55-74 years). In the control group, 20 patients (11 males and 9 females; mean age, 65.9 years; age range, 52-73 years) with unstable ITF were treated using a traditional PFNA technique with the reduction and PFNA implantation under fluoroscopic guidance. All patients were treated by the same surgical team using the same type of PFNA implant (Weigao Orthopedic Device Co., Ltd., Weihai, China). All cases were classified as Müller Arbeitsgemeinschaft für Osteosynthesefragen Classification of fracture types II and III (18). Patients presenting with any of the following were excluded from the present study: Subtrochanteric, pathologic, open, or multiple fractures; isolated fracture of the greater or lesser trochanter; revision hip surgeries; and walking disability before injury caused by paraplegics, severe osteoarthritis, rheumatoid arthritis, lumbar stenosis or lumbar disc herniation. There were no significant differences in patient demographics between the 3DP-RP assisted surgery and traditional surgery groups (Table I). Ethical approval for the present study was granted by the Institutional Medical Ethical Committee of Nanjing Medical University, Nanjing Hospital (Nanjing, China). Informed consent was obtained from each patient prior to the current study.

Digital 3D model reconstruction and 3DP-RP model preparation. Anatomical data obtained by computed tomography (CT) scanning (volume thickness, $1 \mathrm{~mm}$, matrix 512x512; SOMATOM Sensation; Siemens AG, Munich, Germany) were transferred to the planning workstation (M3D; Medical Graphics, Gloucester, UK) using a Digital Imaging and Communications in Medicine interface and a 3D upper femoral model was reconstructed on the screen (Fig. 1). The surgeon then performed the planning using a preoperative planning program (MedGraphics 5.0; MGC Diagnostics, Saint Paul, MN, USA). The software allowed the surgeon to 'navigate' the prosthetic components into the correct positions following fracture reduction using computer aided design (CAD;

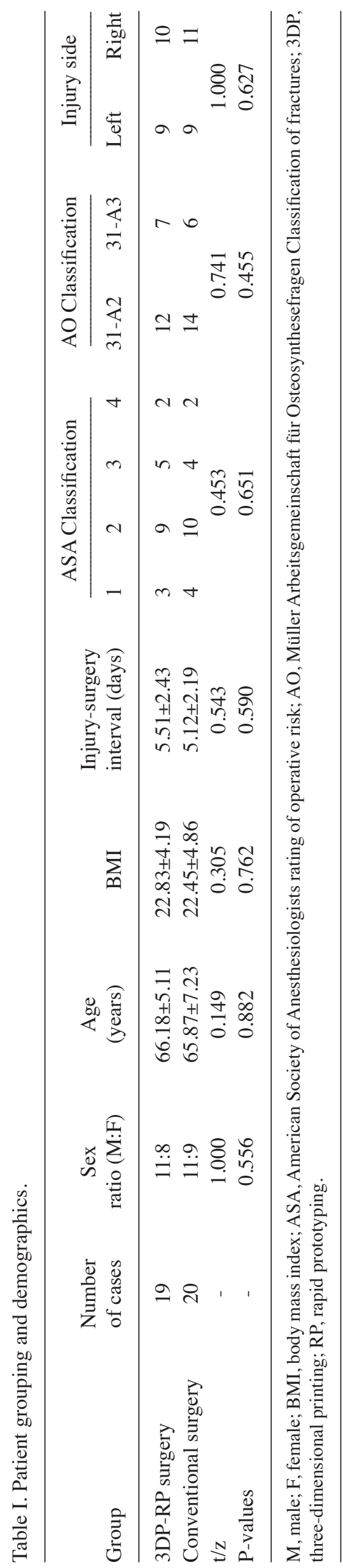



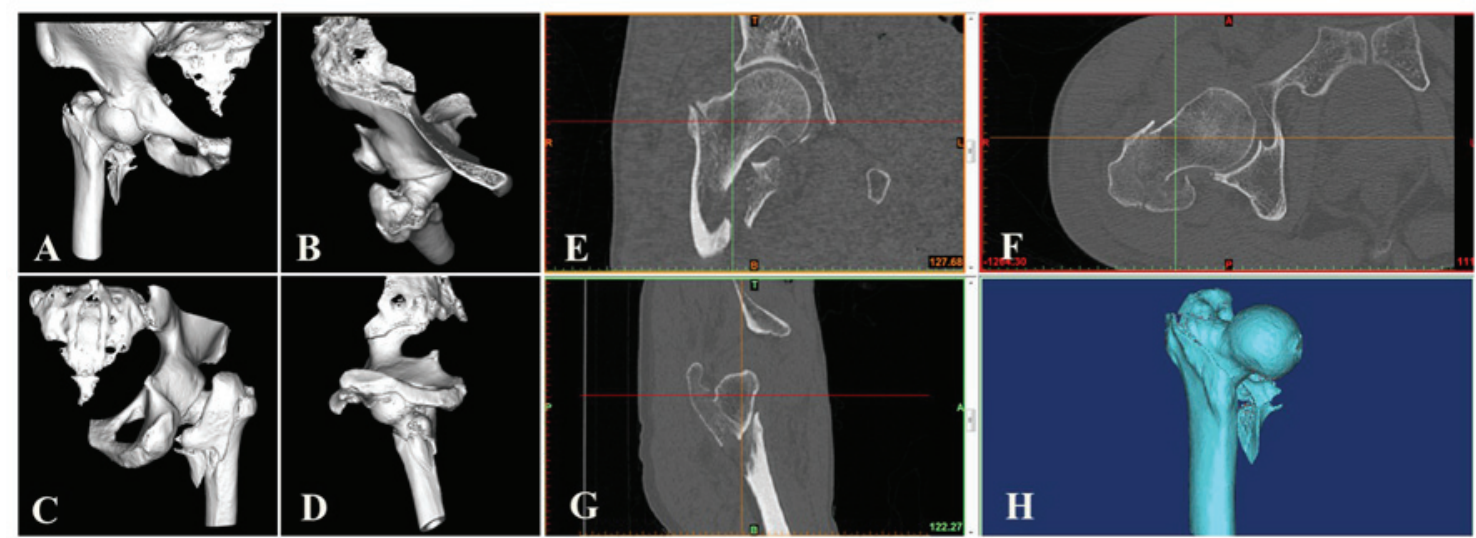

Figure 1. Digital 3D model reconstruction and 3DP-RP model preparation. 3D reconstruction of the intertrochanteric fracture; (A) anterior view, (B) dorsal view, (C) posterior view and (D) ventral view. Multi-planar reconstruction of the intertrochanteric fracture in the (E) coronal, (F) sagittal and (G) transverse planes. (H) 3D model of the same case was obtained by region segmentation and region growing using M3D MedGraphics medical software. 3DP, three-dimensional printing; RP, rapid prototyping.
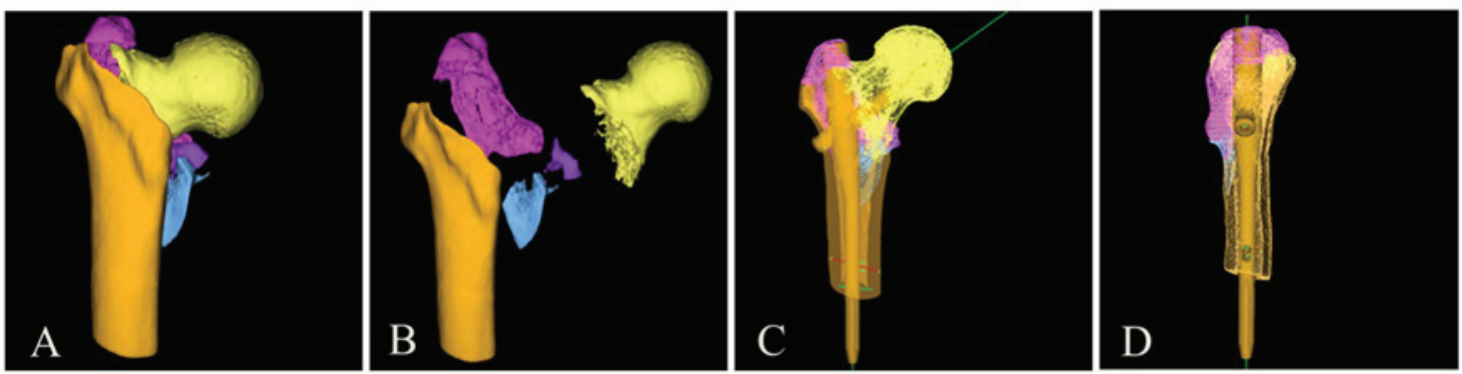

Figure 2. Preoperative planning using CAD. (A and B) A comminuted intertrochanteric fracture as observed by CAD, with fragments marked with different colors. Following simulated reduction of the fracture, the size measurement and prosthetic implant simulation was performed with insert location calculated in the (C) anterior-posterior view and (D) lateral view. CAD, computer assisted design.
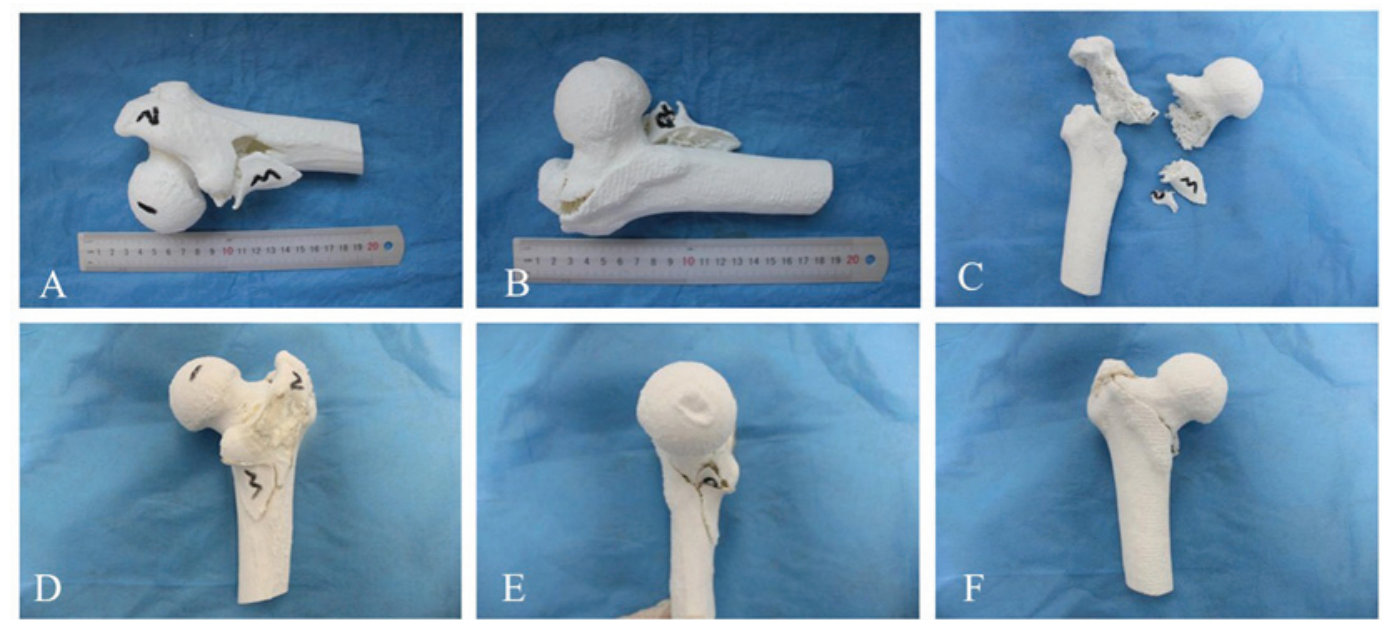

Figure 3. 3DP-RP assisted surgical preparation. A proximal femur model was created based on stereolithography data measuring the size of each fragment; (A) anterior view, (B) posterior view and (C) anterior view of the fragments. Simulated reduction and measurement using the 3DP-RP model; (D) posterior view, (E) sagittal view and (F) anterior view. 3DP-RP, 3D printing rapid prototyping.

Boholo 5.0; Boholo, Shanghai, China) (Fig. 2). The surgeon selected the appropriate sizes of components and recorded them. This model was stored as a stereolithography (STL) file, which was subsequently sent to a 3D printer (Songsun N2, Songsun Medical Technology Co., Ltd., Nanjing, China). The STL file stored the information of actual size, as a 1:1 scale was achieved by CT scanning, due to the design of the scanner and software. The printing procedure was repeated layer by layer until the life-size 3D model was completed (Fig. 3A-C). Simulated fracture reduction was conducted on the model with the femoral neck-shaft angle, size and displacement of fragments measured preoperatively. 
Table II. PFNA parameters at preoperative planning and during surgery.

\begin{tabular}{lcccc}
\hline Parameter & Preoperative planning & Surgery & t-value & P-value \\
\hline Nail diameter $(\mathrm{mm})$ & $9.43 \pm 0.71$ & $9.62 \pm 0.69$ & -0.85 & 0.40 \\
Nail length $(\mathrm{mm})$ & $178.40 \pm 13.84$ & $178.97 \pm 19.27$ & -0.11 & 0.92 \\
Screw blade length $(\mathrm{mm})$ & $85.36 \pm 6.01$ & $86.25 \pm 7.53$ & -0.41 & 0.69 \\
TAD $(\mathrm{mm})$ & $24.07 \pm 0.98$ & $24.18 \pm 1.04$ & 0.34 & 0.74 \\
\hline
\end{tabular}

All data are presented as the mean \pm standard deviation. PFNA, proximal femoral nail anti-rotation; TAD, tip-apex distance.

Table III. Comparison of operating time, intraoperative blood loss, postoperative drainage volume, duration of hospital stay between 3DP group and the conventional surgery group.

\begin{tabular}{lccrr}
\hline & 3DP group & Conventional surgery group & t-value & P-value \\
\hline Operating time (min) & $46.27 \pm 6.51$ & $60.58 \pm 11.92$ & -4.62 & $<0.01$ \\
Intraoperative blood loss (ml) & $98.35 \pm 4.76$ & $162.57 \pm 63.28$ & -4.41 & 0.02 \\
Postoperative blood loss (ml) & $48.63 \pm 4.16$ & $69.18 \pm 15.85$ & -5.48 & 0.03 \\
Time to ambulation (days) & $4.81 \pm 2.23$ & $6.75 \pm 2.64$ & -2.47 & 0.02 \\
Duration of hospital stay (days) & $12.75 \pm 2.49$ & $11.52 \pm 3.14$ & 1.35 & 0.19 \\
\hline
\end{tabular}

All data are presented as the mean \pm standard deviation. ${ }^{\mathrm{a}}<0.05$; $3 \mathrm{DP}, 3 \mathrm{D}$ printing.
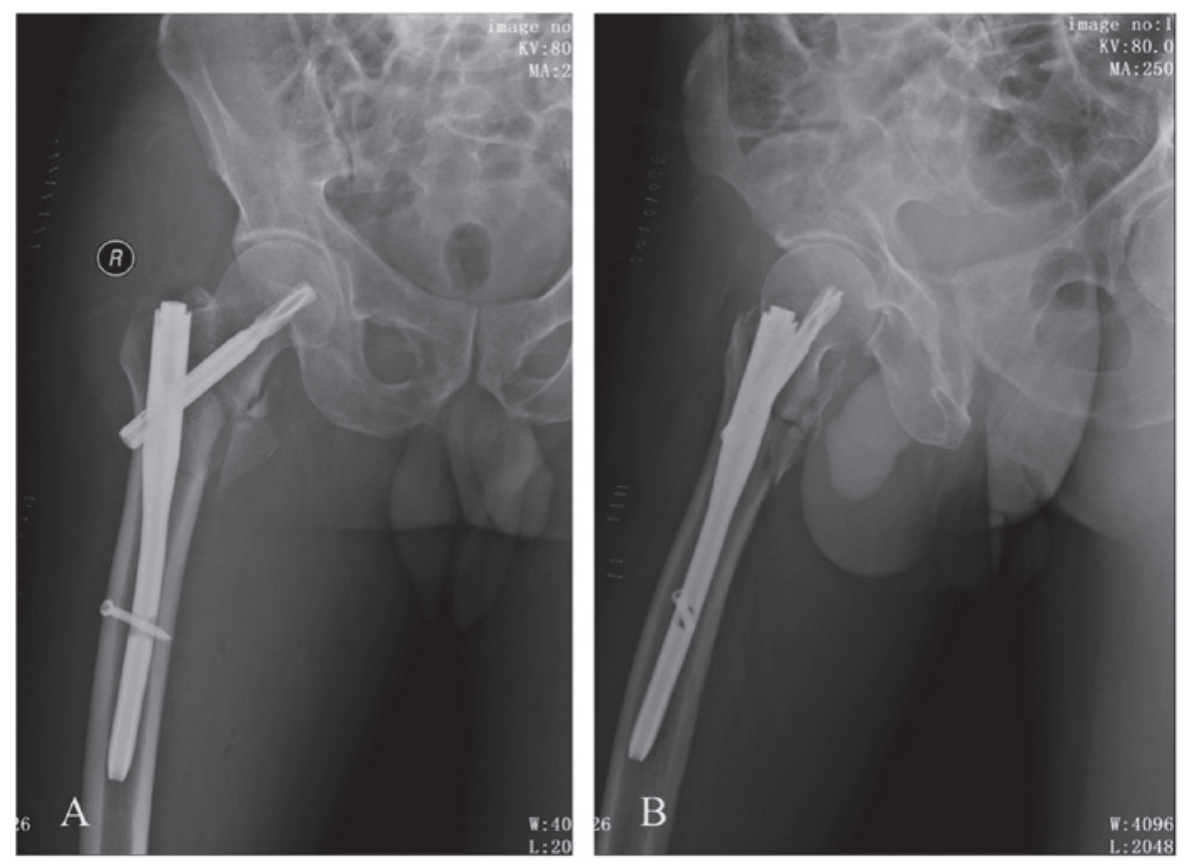

Figure 4. Radiograph image captured 3 days post-surgery. (A) Anterior and (B) lateral views revealed satisfactory reduction and stable internal fixation.

3DP-RP assisted surgical preparation and operative technique. Fracture reduction in the 3DP-RP model allowed simulation of the procedure under direct vision (Fig. 3D-F); optimum reduction ensures good contact and apposition of the internal and posterior cortical bone. The nail and screw blade selected by the M3D software were checked to ensure that the screw blade was located in the ideal position on the femoral head and neck (i.e., central or inferior on the anteroposterior radiograph and central on the lateral radiograph). The tip-apex distance (TAD) was measured using a standard method (19). In the conventional surgery group, the preoperative traction for fracture reduction was performed using the same traction devices under fluoroscopic guidance (OEC FlexiView 8800, GE Healthcare, Chicago, IL, USA).

Next, each fracture reduction and implantation of the PFNA in the two group was performed on a traction table 

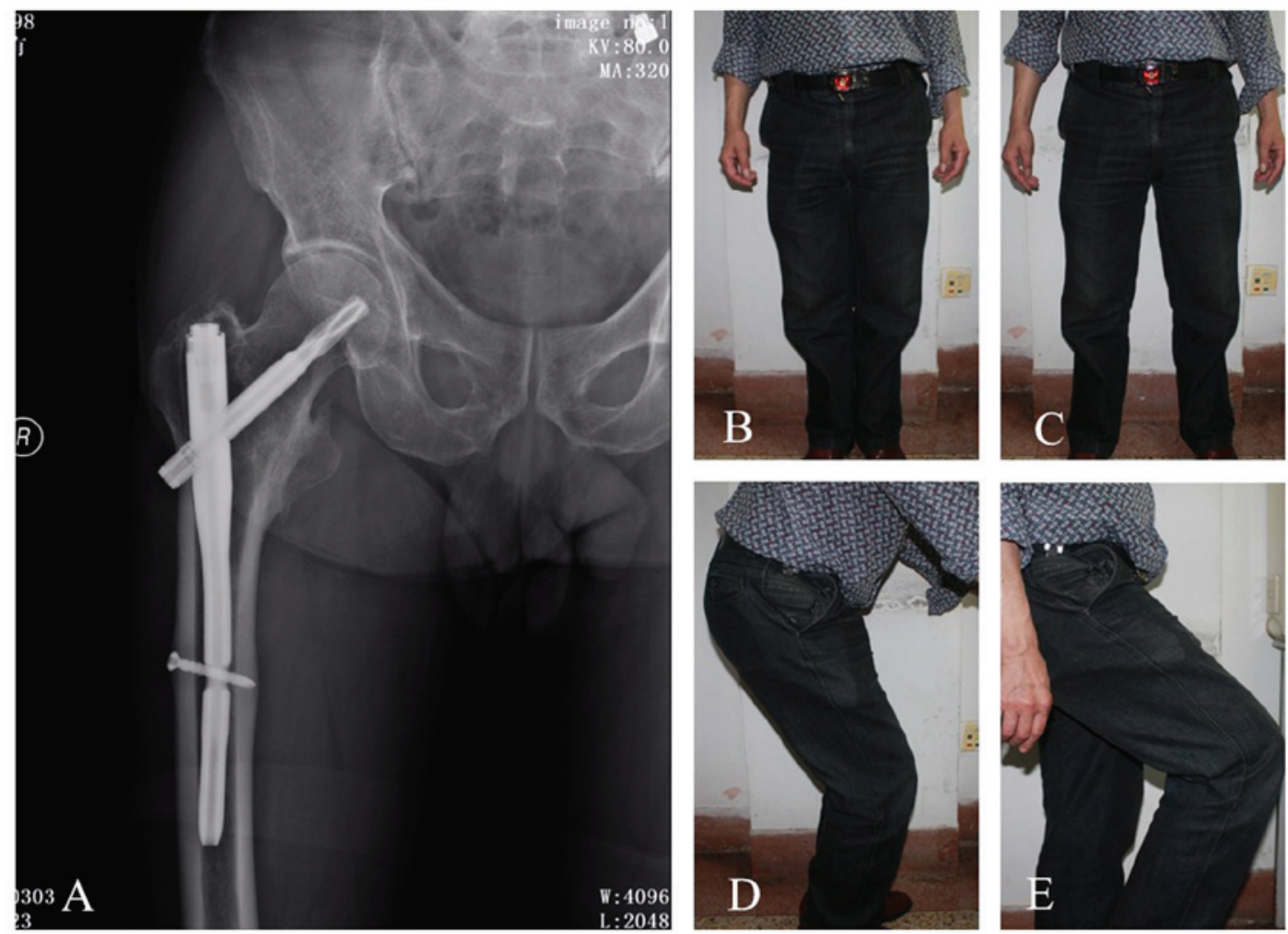

Figure 5. Radiograph image of fracture healing and functional position of the hip joint at the last follow-up of a representative 71-year-old male patient in the 3DP-RP group. (A) X-Ray of fracture healing. (B-E) Hip function was determined to be good at the last follow-up.

under the same fluoroscopic guidance with the orientation of traction designed based on the CAD data and the 3DP-RP model. All tractions were performed under general anesthesia by continuous intravenous injection of propofol $(4-12 \mathrm{mg} / \mathrm{kg} / \mathrm{h}$; Fresenius SE \& Co., Bad Homburg, Germany).

A $5 \mathrm{~cm}$ skin incision was made, the fascia and gluteus medius were isolated and a $3.2 \mathrm{~mm}$ guide wire was inserted from the trochanteric peak. With the soft tissue protected, the proximal femur was manually opened using a bone reamer. The appropriate nail was selected and manually introduced into the femur. Subsequently, the guide wire for the screw blade was inserted through a $2 \mathrm{~cm}$ lateral incision, ensuring the central position in the femoral head in anterior-posterior and lateral fluoroscopic views (20). Final distal locking in the two groups were performed by the navigation of manufacturer's supplementary guiding instruments and fluoroscopy (21).

Postoperative management. All patients received antibiotic prophylaxis (2.0 g twice a day, intravenous; cefathiamidine, Baiyunshan Pharmaceutical Co., Ltd., Guangzhou, China) for $24 \mathrm{~h}$. For thromboprophylaxis, low molecular weight heparin (2,500 U once a day, hypodermic; Fraxiparine, GlaxoSmithKline Co., Ltd., Tianjin, China) was administered subcutaneously until the patients recovered to full mobilization. Supervised weight bearing was allowed as tolerated.

Outcome measures. The parameters of PFNA measured according to the 3DP model, including nail diameter, nail length, screw blade length and TAD, were compared with the actual values obtained during surgery (Table I). Duration of surgery, intraoperative blood loss, postoperative drainage volume and length of hospital stay were recorded and compared with the corresponding values in control patients undergoing conventional PFNA procedures. The volume of postoperative blood loss was calculated as the sum of blood collected in the gauzes (size of each gauze: $45 \times 26 \mathrm{~cm}$, blood volume in each gauze calculated as $15 \mathrm{ml}$ ). Patients were reviewed clinically and radiologically at 3 days post-surgery and followed up for 6 months, with physical evaluations and hip X-rays performed at 1, 3 and 6 months post-surgery. All patients were followed up successfully and no patients were lost.

Statistical analysis. All data are presented as the mean \pm standard deviation. Fisher's exact test and the Student's t-test were used to compare data. Comparison of variables between the groups was performed using the independent sample t test. $\mathrm{P}<0.05$ was considered to indicate a statistical significant difference. SPSS version 13.0 software (SPSS Inc., Chicago, IL, USA) was used for statistical analysis.

\section{Results}

Accuracy of 3DP-DP technology. For the 19 patients in the 3DP-RP group, preoperative planning was performed using CAD and 3DP technology. During surgery, the operative approach, the diameter and length of the nail, the length and position of the screw blade were all consistent with the preoperative plan (Table II).

Comparison of the effects on surgery. Surgical duration, intraoperative and postoperative blood loss were significantly lower in the 3DP-RP group compared with the patients undergoing 
conventional surgery $(\mathrm{P}<0.01, \mathrm{P}=0.02$ and $\mathrm{P}=0.03$, respectively; Table III). There was no significant difference between the two groups in the mean hospital stay duration. At review 3 days post-surgery, radiography revealed satisfactory reductions of all fractures in the two groups, with the implants positioned correctly (Fig. 4). The postoperative follow-up indicated that patients in the 3DP-RP group had a shorter time to ambulation compared with patients that underwent the conventional procedure (4.8 \pm 2.2 vs. $6.7 \pm 2.6$ days; $\mathrm{P}<0.05$; Table II).

Follow-up clinical outcome analysis. At the 6-month follow-up, bone healing was satisfactory in all cases in the two groups, without the screw cut out of the head or obvious displacement of fracture segments. All patients in the two groups exhibited good hip function with no varus/vague hip deformities observed (Fig. 5).

\section{Discussion}

ITF is common in elderly people aged $>65$ years, with a particularly high incidence in individuals aged $>70$ years (21). Due to a significant loss of bone mass in elderly patients, the femoral head and neck may not require fixation, thus selecting the best regions for PFNA internal fixation is of particular importance. Baumgaertner et al (22) reported that when the TAD is $<25 \mathrm{~mm}$, there is a significantly lower risk of the femoral head cutting out and fixation is more reliable. In the present study, the 3DP-RP technique facilitated accurate preoperative planning, resulting in satisfactory internal fixation and TAD within a reasonable range. None of the patients enrolled in the present study exhibited varus/vague hip deformities, screw cutouts or femoral shaft fractures at the 6-month follow-up.

Perfect traction and reduction with good contact of the interior and posterior cortical bone are very important in ITF correction to reduce the risk of varus hip deformity developing later on $(23,24)$. Fracture reduction has previously relied on preoperative radiography and intraoperative fluoroscopy (25). In the present study, using the 3DP-RP prototype made it possible to fully characterize the $3 \mathrm{D}$ structure of the ITF and the positional relationship among the fracture fragments preoperatively. Therefore, fractures were classified more accurately and traction reduction was completed more rapidly. Indeed, the duration of 3DP-RP assisted surgery was significantly lower than that of traditional surgery. In the present study, information derived from the use of 3DP-RP technology improved internal fixation and contributed during the preoperative application of traction.

The results of the present study demonstrate that, compared with traditional surgery, the use of 3DP-RP technology has marked advantages in PFNA surgery as it reduced the duration of surgery, as well as intraoperative and postoperative blood loss. The postoperative follow-up indicated that the time to early ambulation may also be shorter.

The present study, as well as results from previous studies $(14,26)$, identified certain limitations in the current use of 3DP-RP assisted PFNA for the treatment of ITF. Although it could aid surgical procedure through the reconstruction of precise anatomical structure, the 3DP-RP technique in the current study was not able to produce an accurate percutaneous navigation template for the locking screw implantation during the surgery, particularly the distal locking nail implantation. In the present study, 3DP-RP was unable to penetrate the soft tissue, therefore accurate mechanical analysis was not possible and it was necessary to rely on the experience of the surgeon and intraoperative C-arm fluoroscopy to produce traction reduction. At present, the data acquisition and processing times for 3DP model printing are between 48 and $72 \mathrm{~h}$. The requirement for specialized software and hardware and specially trained personnel increases medical expenses; however, these limitations are likely to be minimized as 3DP-RP technology matures and becomes less expensive. Since the current study cannot be classed as a blinded trial, further research will be necessary to observe the effect of 3DP-RP surgery in a larger, blinded prospective study.

In conclusion, the use of the 3DP-RP model prior to internal fixation in ITF allows complete preoperative planning and better intraoperative orientation of risk structures, thus improving the efficiency of the surgical procedure and patient outcomes.

\section{Acknowledgements}

The present study was supported by the Key Research Program of Science and Technology of Jiangsu Province (grant nos. BE 2015613, BE 2016763).

\section{References}

1. Moran CG, Wenn RT, Sikand M and Taylor AM: Early mortality after hip fracture: Is delay before surgery important? J Bone Joint Surg Am 87: 483-489, 2005.

2. Dhanwal DK, Dennison EM, Harvey NC and Cooper C: Epidemiology of hip fracture: Worldwide geographic variation. Indian J Orthop 45: 15-22, 2011.

3. Roche JJ, Wenn RT, Sahota O and Moran CG: Effect of comorbidities and postoperative complications on mortality after hip fracture in elderly people: Prospective observational cohort study. BMJ 331: 1374, 2005.

4. Shen L, Zhang Y, Shen Y and Cui Z: Antirotation proximal femoral nail versus dynamic hip screw for intertrochanteric fractures: A meta-analysis of randomized controlled studies. Orthop Traumatol Surg Res 99: 377-383, 2013.

5. Yu W, Zhang X, Zhu X, Hu J and Liu Y: A retrospective analysis of the InterTan nail and proximal femoral nail anti-rotation-Asia in the treatment of unstable intertrochanteric femur fractures in the elderly. J Orthop Surg Re 11: 10, 2016.

6. Queally JM,Harris E, Handoll HH and Parker MJ: Intramedullary nails for extracapsular hip fractures in adults. Cochrane Database Syst Rev 12: CD004961, 2014.

7. Mereddy P, Kamath S, Ramakrishnan M, Malik H and Donnachie N: The AO/ASIF proximal femoral nail antirotation (PFNA): A new design for the treatment of unstable proximal femoral fractures. Injur 40: 428-432, 2009.

8. Simmermacher RK, Ljungqvist J, Bail H, Hockertz T, Vochteloo AJ, Ochs U and Werken Cv; AO-PFNA studygroup: The new proximal femoral nail antirotation (PFNA) in daily practice: Results of a multicentre clinical study. Injury 39: 932-939, 2008.

9. Garg B, Marimuthu K, Kumar V, Malhotra R and Kotwal PP: Outcome of short proximal femoral nail antirotation and dynamic hip screw for fixation of unstable trochanteric fractures. A randomised prospective comparative trial. Hip Int 21: 531-536, 2011.

10. Xu YZ, Geng DC, Mao HQ, Zhu XS and Yang HL: A comparison of the proximalfemoral nail antirotation device and dynamic hip screw in the treatment of unstable pertrochanteric fracture. J Int Med Res 38: 1266-1275, 2010.

11. Pu JS, Liu L, Wang GL, Fang Y and Yang TF: Results of the proximal femoral nail anti-rotation (PFNA) in elderly Chinese patients. Int Orthop 33: 1441-1444, 2009. 
12. Costa ML, Griffin XL, Achten J, Metcalfe D, Judge A, Pinedo-Villanueva $R$ and Parsons N: World Hip Trauma Evaluation (WHiTE): Framework for embedded comprehensive cohort studies. BMJ Open 6: e011679, 2016.

13. Yang YH, Wang YR, Jiang SD and Jiang LS: Proximal femora nail antirotation and third-generation Gamma nail: Which is a better device for the treatment of intertrochanteric fractures? Singapore Med J 54: 446-450, 2013.

14. Chana-Rodríguez F, Mañanes RP, Rojo-Manaute J, Gil P, Martínez-Gómiz JM and Vaquero-Martín J: 3D surgical printing and pre contoured plates for acetabular fractures. Injury 47: 2507-2511, 2016

15. Suh YS, Nho JH, Kim SM, Hong S, Choi HS and Park JS: Clinical and radiologic outcomes among bipolar hemiarthroplasty, compression hip screw and proximal femur nail antirotation in treating comminuted intertrochanteric fractures. Hip Pelvis 27: $30-35,2015$.

16. Zhang YZ, Lu S, Zhang HQ, Jin ZM, Zhao JM, Huang J and Zhang ZF: Alignment of the lower extremity mechanical axis by computer-aided design and application in total knee arthroplasty. Int J Comput Assist Radiol Surg 11: 1881-1890, 2016.

17. Zhang L, Lv H, Zheng H, Li M, Yin P, Peng Y, Gao Y, Zhang L and Tang P: Correlation between parameters of calcaneal quantitative ultrasound and hip structural analysis in osteoporotic fracture patients. PLoS One 10: e0145879, 2015.

18. Urrutia J, Zamora T, Besa P, Zamora M, Schweitzer D and Klaber I: Inter and intra-observer agreement evaluation of the $\mathrm{AO}$ and the Tronzo classification systems of fractures of the trochanteric area. Injury 46: 1054-1058, 2015.

19. Knobe M, Drescher W, Heussen N, Sellei RM and Pape HC: Is helical blade nailing superior to locked minimally invasive plating in unstable pertrochanteric fractures? Clin Orthop Relat Res 470: 2302-2312, 2012.
20. Vidyadhara S and Rao SK: One and two femoral neck screws with intramedullary nails for unstable trochanteric fractures of femur in the elderly-randomised clinical trial. Injury 38: 806-814, 2007.

21. Li M, Wu L, Liu Y and Wang C: Clinical evaluation of the Asian proximal femur intramedullary nail antirotation system (PFNA-II) for treatment of intertrochanteric fractures. J Orthop Surg Res 9: 112, 2014.

22. Baumgaertner MR, Curtin SL, Lindskog DM and Keggi JM: The value of the tip-apex distance in predicting failure of fixation of peritrochanteric fractures of the hip. J Bone Joint Surg Am 77: 1058-1064, 1995.

23. Cooper C, Cole ZA, Holroyd CR, Earl SC, Harvey NC, Dennison EM, Melton LJ, Cummings SR and Kanis JA; IOF CSA Working Group on Fracture Epidemiology: Secular trends in the incidence of hip and other osteoporotic fractures. Osteoporos Int 22: 1277-1288, 2011

24. Audigé L, Cagienard F, Sprecher CM, Suhm N and Müller MA: Radiographic quantification of dynamic hip screw migration. Int Orthop 38: 839-845, 2014.

25. Herman A, Landau Y, Gutman G, Ougortsin V, Chechick A and Shazar N: Radiological evaluation of intertrochanteric fracture fixation by the proximal femoral nail. Injury 43: 856-863, 2012.

26. Lv H, Zhang L, Yang F, Li M, Yin P, Su X, Yin P, Zhang L and Tang P: A novel 3D-printed device for localization and extraction of trabeculae from human femoral heads: A comparison with traditional visual extraction. Osteoporos Int 26: 1791-1799, 2015. 